\title{
Zero-point vibrational corrections to isotropic hyperfine coupling constants in polyatomic molecules
}

\author{
Xing Chen, ${ }^{a b}$ Zilvinas Rinkevicius, ${ }^{b}$ Zexing Cao, ${ }^{a}$ Kenneth Ruud $^{c}$ and Hans Ågren ${ }^{b}$ \\ Received 6th August 2010, Accepted 7th October 2010 \\ DOI: $10.1039 / \mathrm{c0cp01443e}$
}

The present work addresses isotropic hyperfine coupling constants in polyatomic systems with a particular emphasis on a largely neglected, but a posteriori significant, effect, namely zero-point vibrational corrections. Using the density functional restricted-unrestricted approach, the zero-point vibrational corrections are evaluated for the allyl radical and four of its derivatives. In addition for establishing the numerical size of the zero-point vibrational corrections to the isotropic hyperfine coupling constants, we present simple guidelines useful for identifying hydrogens for which such corrections are significant. Based on our findings, we critically re-examine the computational procedures used for the determination of hyperfine coupling constants in general as well as the practice of using experimental hyperfine coupling constants as reference data when benchmarking and optimizing exchange-correlation functionals and basis sets for such calculations.

\section{Introduction}

Electronic struture theory in general, and density functional theory (DFT) in particular, has in recent years evolved to become a sophisticated modeling approach capable of determining electronic g-tensors, ${ }^{1-10}$ hyperfine coupling constants ${ }^{8,9,11-18}$ and zero-field splitting parameters ${ }^{19-25}$ of a variety of paramagnetic compounds. Despite the significant progress witnessed in this area, the capability of DFT to accurately predict these quantities beyond the domain of organic radicals are still under debate and can at present only be considered semiquantitatively or qualitatively correct for the most important classes of systems such as paramagnetic transition metal complexes. ${ }^{4,9,14,26-28}$ Among the electron paramagnetic resonance (EPR) spin Hamiltonian parameters, the hyperfine coupling constants (HFCCs) are the most problematic to calculate, as they require a computational approach that must be capable of accurately describing the electron density distribution in regions near the magnetic nuclei, and at the same time be capable of accounting for the conformational, environmental and vibrational effects. ${ }^{26,29}$ Due to the inherit complexity of procedures for calculating the latter effects, the strict evaluation of HFCCs has remained limited to small, mostly organic, radicals with few atoms, and so far only a handful of such studies have been carried out ${ }^{29-33}$ and the first results were presented only recently by Barone et al. ${ }^{34-39}$ In order to cope with the computational demands for larger radicals, the vibrational effects on the HFCCs are usually neglected entirely ${ }^{40-43}$ or accounted for using a single large amplitude motion model. ${ }^{36,44-46}$ This follows from the fact that a straightforward numerical evaluation of the full vibrational

\footnotetext{
${ }^{a}$ Department of Chemistry and State Key Laboratory of Physical Chemistry of Solid Surfaces, Xiamen University, Xiamen 361005, China

${ }^{b}$ Department of Theoretical Chemistry, School of Biotechnology, Royal Institute of Technology, SE-106 91 Stockholm, Sweden ${ }^{c}$ Centre for Theoretical and Computational Chemistry, Department of Chemistry, University of Tromso, N-9037 Tromso, Norway
}

corrections to HFCCs, with both zero-point and finite temperature contributions, becomes unfeasible already for mediumsize polyatomic molecules. The strategy outlined for the determination of HFCCs, in which all important effects are accounted for except for the vibrational corrections, are widely accepted and have been applied quite extensively to various organic radicals ${ }^{40-43}$ and several transition metal complexes. ${ }^{9,14}$ The notion that vibrational effects on HFFCs are overall insignificant in practical calculations, except from special cases such as the methyl radical, ${ }^{47}$ has become common practice and most investigations of HFCCs have thus until now been carried out without taking vibrational effects into consideration.

The vibrational contributions to hyperfine coupling constants can be separated into two distinct types; zero-point and temperature dependent vibrational corrections, where the former arises from the vibrational part of the total wave function at zero temperature and the latter refers to an ensemble of molecular excited vibrational states occupied at the temperature of measurement and with corrections due to centrifugal distortions arising from the population of higher rotational states. The size of the temperature-dependent vibrational corrections can be deduced from a variable temperature or/and isotope substitution EPR experiments, but while many examples of such measurements have been presented over the years ${ }^{48-51}$ they are severely hampered by the stability of radicals over the typical range of temperatures required in the measurements. The extraction of "pure" HFCCs corresponding to zero temperature has consequently remained limited to selected stable radicals. The lack of extensive experimental data has restricted the development of empirical guidelines for a priori identification of radicals in which a significant temperature-dependent vibrational correction to the HFCCs can be expected. It is clear that both theoretical and experimental investigations of temperature-dependent vibrational corrections to HFFCs are warranted in order to establish their importance.

The estimation of zero-point vibrational corrections (ZPVCs) to HFCCs is even more complicated, since the 
ZPVCs cannot be extracted directly from experiment and must rather rely on theoretical calculations only. Unfortunately, only a limited number of computational results for zero-point vibrational corrections to HFCCs are currently available ${ }^{29-39}$ and this is clearly insufficient for making any conclusion on their importance for even very specific classes of organics radicals. Thus, new systematic studies of ZPVCs to HFCCs for general organic radicals and paramagnetic transition metal complexes are called for in order to advance our understanding of their importance.

In this work we take the first step towards a systematic investigation of zero-point vibrational corrections to hyperfine coupling constants, using hydrogens in allylic radicals as a case study. In addition to numerically evaluating the ZPVCs, we also formulate guidelines for identifying the cases in which ZPVCs to HFCCs of hydrogens can be expected to be significant. These guidelines will hopefully aid in computations of hydrogen HFCCs in other organic radicals as well as more rigorous interpretations of experimental results. In the following, we briefly describe the computational methodology used to evaluate ZPVCs to isotropic HFCCs in polyatomic radicals and present the results for the allyl radical and four of its derivatives. We conclude by presenting the guidelines for identifying hydrogens with large ZPVCs for the isotropic HFCCs and critically re-examine the computational procedure used for determining HFCCs in view of these new findings.

\section{Zero-point vibrational corrections to hyperfine coupling constants: computational strategy for polyatomic radicals}

The isotropic hyperfine coupling constants of protons as well as of other light magnetic nuclei are almost exclusively defined by the Fermi contact (FC) interaction between the spin of the magnetic nucleus and the spins of the unpaired electrons in the paramagnetic molecule. ${ }^{26,29}$ The task of computing these constants can be readily accomplished with the help of first order perturbation theory and for DFT methods that are variational with respect to triplet perturbations, this approach reduce to a straightforward evaluation of an expectation value of the FC operator. ${ }^{26,29}$ Therefore, in DFT, the isotropic HFCCs are mostly evaluated using the unrestricted Kohn-Sham (UKS) formalism, ${ }^{11,12,14,15}$ in which the advantage of the stability of an unrestricted energy functional with respect to triplet perturbations is exploited. Despite the inherit simplicity of this computational procedure in the unrestricted formalism, it suffers from problems due to spin contamination of the unrestricted Kohn-Sham wave functions which introduces unpredictable errors for radicals with a spincontaminated ground state, an issue that in general cannot be controlled in an efficient way. In this work we employ an alternative DFT methodology for the evaluation of HFCCs constants, namely the restricted-unrestricted (DFT-RU) approach, ${ }^{9,16,18}$ which is free from spin-contamination problems. This approach is based on the open-shell spin-restricted Kohn-Sham formalism for the ground state ${ }^{52}$ and on an unrestricted approach for the perturbational treatment of triplet properties. ${ }^{9,16,18}$ In this way, spin-contamination of the ground state is avoided while spin polarization still is included in the property evaluation (here the isotropic HFCCs). ${ }^{9,16,18}$ In the DFT-RU approach, the isotropic hyperfine coupling constant of the $n$-th nucleus can be computed as

$$
A_{n}^{i s o}=\left.\left\langle 0\left|\frac{\partial^{2} \hat{H}_{F C}}{\partial \mathscr{S}_{z} \partial I_{z}^{n}}\right| 0\right\rangle\right|_{\mathscr{S}_{z}=0, I_{z}^{n}=0}+\left.\left\langle\left\langle\frac{\partial^{2} \hat{H}_{F C}}{\partial \mathscr{S}_{z} \partial I_{z}^{n}} ; \hat{H}_{0}\right\rangle\right\rangle_{0,0}\right|_{\mathscr{S}_{z}=0, I_{z}^{n}=0},
$$

where $\mathscr{S}_{z}$ is the Cartesian $z$ component of the effective electronic spin, $I_{z}^{n}$ is the Cartesian $z$ component of the nuclear spin of the n-th magnetic nucleus, $\hat{H}_{F C}$ is the FC operator, and $\hat{H}_{0}$ is the zeroth-order Kohn-Sham Hamiltonian of the unperturbed system. In eqn (1), the first term is the conventional expectation value of the FC operator, which describes the direct spin-density contribution to the FC interaction, and the second term is the spin polarization correction to the FC interaction. The latter describes the electron density relaxation in the presence of a triplet perturbation such as the FC operator, and is given by a triplet linear response function following previous work by Rinkevicius et al..$^{9,16}$ Therefore, in the DFT-RU approach, the direct spin-density and spin polarization contributions to the isotropic HFCCs are strictly separated and a rigorous analysis of each contribution is possible. These advantages of the DFT-RU method have already been exploited in studies of HFCCs of organic radicals. ${ }^{16,18}$ Due to these features, we will use the DFT-RU approach for the evaluation of zero-point vibrational corrections to HFCCs in this study.

In polyatomic molecules the most convenient strategy for evaluating ZPVCs to molecular properties is to employ perturbation theory for the vibrational wave functions and in this way compute zero-point vibrational corrections up to a desirable order in the perturbation theory expansion. ${ }^{53-55}$ Among these types of approaches, two distinct formalisms are most commonly employed in studies of ZPVCs to molecular properties: in one approach the property is expanded around the equilibrium geometry of the molecule and both harmonic and anharmonic vibrational corrections are computed in a perturbative manner, ${ }^{53,54}$ in the second formalism, the property is expanded around the so-called effective geometry ${ }^{56}$ with the harmonic correction computed as a perturbation, while the anharmonic correction is given by the difference between the property values evaluated at the equilibrium and effective geometries of the molecule, respectively. ${ }^{55}$ In this work, we adopt the second formalism for the evaluation of ZPVCs to the isotropic hyperfine coupling constants, as it has been shown to be the more accurate one in studies of ZPVCs to properties of diatomic molecules, see the work Astrand, Ruud and Sundholm. ${ }^{57}$ Within the second formalism, the zero-point vibrationally corrected isotropic hyperfine coupling constant of nucleus $n$ can be written as

$$
A_{n, \mathrm{vib}}^{\text {iso }}=A_{n, \mathrm{eff}}^{\text {iso }}+\frac{1}{4} \sum_{K} \frac{1}{\omega_{K}} \frac{\partial^{2} A_{n, \text { eff }}^{\text {iso }}}{\partial Q_{K}^{2}},
$$

where $A_{n \text {,eff }}^{\text {iso }}$ is the isotropic hyperfine coupling constant evaluated at the effective geometry, $\partial^{2} A_{n \text {,eff }}^{\text {iso }} / \partial Q_{K}^{2}$ is the second derivative of $A_{n \text {,eff }}^{\text {iso }}$ with respect to normal mode $Q_{K}, \omega_{K}$ is the 
<smiles></smiles>

$\left(\mathrm{C}_{2 \mathrm{~V}},{ }^{2} \mathrm{~A}_{2}\right)$ Allyl

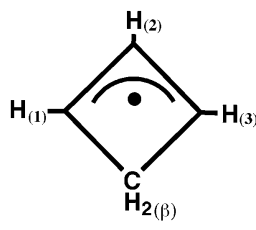

$\left(\mathrm{C}_{2 \mathrm{~V}},{ }^{2} \mathrm{~A}_{2}\right)$

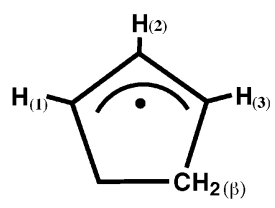

$\left(\mathrm{C}_{2 \mathrm{~V}},{ }^{2} \mathrm{~A}_{2}\right)$

Cyclopentenyl

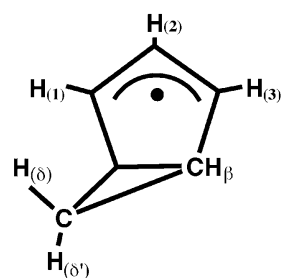

$\left(\mathrm{C}_{\mathrm{s}},{ }^{\mathbf{2}} \mathrm{A}^{\prime \prime}\right)$

Bicyclo[3.1.0]hexenyl

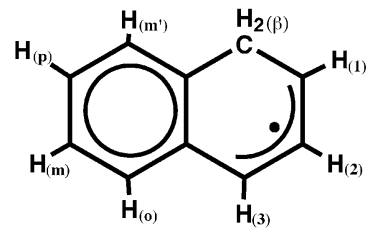

$\left(\mathrm{C}_{\mathrm{s}},{ }^{2} \mathrm{~A}^{\prime \prime}\right)$

1-Hydronaphthyl

Fig. 1 The five allylic radicals investigated in this work: allyl, cyclobutenyl, cyclopentenyl, bicyclo[3.1.0]hexenyl and 1-hydronaphthyl.

corresponding $K^{\prime}$ th vibrational frequency computed at the effective geometry, and the summation index $K$ runs over all vibrational modes of the molecule, i.e. over $(3 N-6)$ modes for non-linear and $(3 N-5)$ modes for linear molecules, $N$ being the number of atoms in molecule. In the above equation, we can readily identify the first term as the sum of the isotropic HFCCs at the equilibrium geometry $A_{n \text {,eql }}^{\text {iso }}$ and its anharmonic zeropoint vibrational correction $A_{n, \mathrm{anh}}^{\text {iso }}$, i.e. $A_{n \text {, fff }}^{\text {iso }}=A_{n \text {, eq } 1}^{\text {iso }}+A_{n, \text { anh }}^{\text {iso }}$, and the second term as the harmonic zero-point vibrational correction $A_{n \text {,harm }}^{\text {iso }}$ to $A_{n \text {,eff }}^{\text {iso }}$, respectively. From these two ZPVCs to the isotropic HFCC, the evaluation of the latter correction is more demanding computationally as it requires the determination of the geometric Hessian and the second derivatives of $A_{n, \text { eff }}^{\text {iso }}$ with respect to the vibrational modes at the effective geometry, while the evaluation of the former correction is rather straightforward and requires only an isotropic HFCC computation at the equilibrium and effective geometries. In addition to these computations, the calculation of ZPVCs to the isotropic HFCCs also requires a determination at the effective geometry of the molecule, ${ }^{56}$ which can be obtained from a calculation at the equilibrium geometry $\left\{R_{\mathrm{eql}, K}\right\}$ in the following manner

$$
\left\{R_{\mathrm{eff}, K}\right\}=\left\{R_{\mathrm{eql}, K}\right\}-\frac{1}{4 \omega_{K}^{2}} \sum_{L} \frac{V_{\mathrm{eql}, K L L}^{(3)}}{\omega_{L}},
$$

where $V_{\mathrm{eql}, K L L}^{(3)}$ is the semidiagonal elements of the cubic force field at the equilibrium geometry. In short, our evaluation of ZPVCs to HFCCs consists of fours steps: (1) determination of the effective geometry; (2) evaluation of the anharmonic zero-point vibrational correction to $A_{n \text {,eql }}^{\text {iso }}$ (3) determination of the geometric Hessian at the effective geometry; and (4) evaluation of the harmonic ZPVC contribution to $A_{n \text {,eff. The }}^{\text {iso }}$ strategy outlined for the evaluation of ZPVCs to HFCCs is computationally more demanding compared to the more frequently used approach, which is based on an expansion of the molecular property around the equilibrium geometry instead of the effective geometry. However, in many cases the numerical evaluation of $\partial^{2} A_{n \text {,eff }}^{\text {iso }} / \partial Q_{K}^{2}$ derivatives forms the dominant computational step in the whole procedure, and, in our opinion, the gains in accuracy obtained by expanding the isotropic HFCCs around the effective geometry instead of the equilibrium geometry outweighs the additional computation cost of the former. In this work, we therefore employ the methodology based on the effective geometry for evaluating the ZPVCs to the isotropic hyperfine coupling constants.

\section{Computational details}

In order to illustrate our methodology for computing vibrational corrections to HFCCs, we investigate in detail one specific class of $\pi$-type organic radicals, namely allylic radicals. The main criteria for the selection of these radicals are that they do not contain any methyl groups or any groups or fragments with shallow double-well potentials, and that their lowest-energy conformations are well separated from other conformations. In addition, experimental EPR measurements have been conducted at low temperature and with an innoccuous environment such as noble gas matrices or liquid alkanes. Following these intial considerations, we have chosen to include five allylic radicals in our test set (see Fig. 1): the allyl radical $\left(\mathrm{C}_{2 \mathrm{v}}\right.$ symmetry, ${ }^{2} A_{2}$ ground state), the cyclobutenyl radical $\left(\mathrm{C}_{2 \mathrm{v}}\right.$ symmetry, ${ }^{2} A_{2}$ ground state $)$, the cyclopentenyl radical $\left(\mathrm{C}_{2 \mathrm{v}}\right.$ symmetry, ${ }^{2} A_{2}$ ground state), the bicyclo[3.1.0]hexenyl radical $\left(\mathrm{C}_{\mathrm{s}}\right.$ symmetry, ${ }^{2} A^{\prime \prime}$ ground state) and the 1-hydronaphtyl radical $\left(\mathrm{C}_{\mathrm{s}}\right.$ symmetry, ${ }^{2} A^{\prime \prime}$ ground state).

Equilibrium and effective geometries of the radicals as well as their geometric Hessians at the effective geometries have been obtained using the spin-restricted open-shell Kohn-Sham formalism, where geometry optimization is performed using analytical geometrical gradients and where the calculation of effective geometries and the geometric Hessian at this geometry uses numerical first and second geometric derivatives. All calculations of the geometrical parameters and the quadratic and cubic fields of the radicals have been performed using the B3LYP exchange-correlation functional ${ }^{58-61}$ and the TZV2P basis set ${ }^{62}$ according to recommendations by Boese et al. ${ }^{63}$ The DFT-RU calculations of the isotropic hyperfine coupling constants at the equilibrium and effective geometries as well as the numerical evaluation of the $\partial^{2} A_{n, \text { eff }}^{\text {iso }} / \partial Q_{K}^{2}$ derivatives, in contrast to the force fields calculations, have been carried out using the HIII-su3 basis set, ${ }^{64-67}$ which ensures an accurate description of the electron density in the inner core as well as the valence regions of the molecule. The selection of the HIII-su3 basis set is motivated by its inherent good accuracy; isotropic HFCCs of hydrogens obtained in this basis set are close to the ones obtained with the larger and computationally considerably more expensive HIV-su4 basis set, ${ }^{64-67}$ which have been used in earlier DFT-RU benchmark studies of organic radicals. ${ }^{16,18}$

After settling the main computational details of the ZPVCs to the isotropic HFCCs, let us now turn to the more subtle aspects of these calculations, namely the numerical evaluation of the quadratic and cubic force fields, and the $\partial^{2} A_{n, \text { eff }}^{\text {iss }} / \partial Q_{K}^{2}$ 
derivatives. We have used a three-point differentiation scheme in all these calculations, where the cubic force field elements and $\partial^{2} A_{n \text {,eff }}^{\text {iso }} / \partial Q_{K}^{2}$ derivatives have been taken along the normal coordinates of the vibrational modes, and where the quadratic force field elements have been computed using Cartesian displacements. In order to ensure numerical stability of this numerical differentiation we used a step size of 0.0075 a.u. as recommended by Ruud, Astrand and Taylor, ${ }^{55}$ and also tightened the convergence thresholds of the iterative solution of the Kohn-Sham and linear response equations to $10^{-9}$ gradient norm and $10^{-7}$ residual norm, respectively. All calculations have been carried out in a parallel fashion using the development version of DALTON quantum chemistry program, ${ }^{68}$ allowing us to perform ZPVCs even for mediumsized organic radicals, having from twenty or more atoms.

\section{Results and discussion}

We will try to address ZPVCs corrections to isotropic HFCCs in general using hydrogens in allylic radicals as a basis for the discussion. We will first focus on the isotropic HFCCs and the impact of ZPVCs on each radical separately before we extract the important factors common to all the ZPVCs of the isotropic HFCCs in the allylic radicals and propose guidelines for identifying hydrogens with significant ZPVCs in these and other $\pi$-type organic radicals.

\section{A. Allyl radical}

Among the radicals investigated in this work, the allyl radical has previously been extensively studied and many works both by experimentalists and theoreticians have been devoted to the hyperfine structure of its EPR spectrum. ${ }^{69-74}$ The isotropic hyperfine coupling constants of both hydrogens and carbons in this radical arise solely from spin polarization effects and follow a specific pattern: the isotropic HFCC of the hydrogen connected to the central carbon is small and positive; the isotropic HFCCs of the hydrogens located on the terminal $-\mathrm{CH}_{2}$ groups are relatively large and negative. The isotropic HFCCs of the hydrogens computed using the DFT-RU approach along with the experimental results of Fessenden et al. ${ }^{69}$ are given in Table 1. Overall, the isotropic HFCCs at the equilibrium geometry, $A_{\text {eql }}^{\text {iso }}$, evaluated using the DFT-RU method are in good agreement with experimental data; deviations do not exceed $\pm 1.0 \mathrm{G}$ and the ordering observed in experiment is reproduced. In this respect, we note that the isotropic HFCCs obtained by Adamo et al. ${ }^{73}$ using the UKS method are almost identical to the ones computed here using the DFT-RU method; the discrepancies between the two methods are about \pm 0.1 G. On the other hand, highly correlated methods, such as coupled cluster with single and double substitutions (CCSD), systematically overestimate, in terms of absolute values, the isotropic HFCCs in the allyl radical relative to experiment and predicts $A_{\mathrm{eq} 1}^{\text {iso }}\left(\mathrm{H}_{(2)}\right)$ to be $5.95 \mathrm{G}$, $A_{\text {eql }}^{\text {iso }}\left(\mathrm{H}_{(1,3)}^{\text {exo }}\right)$ to $-18.50 \mathrm{G}$ and $A_{\text {eql }}^{\text {iso }}\left(\mathrm{H}_{(1,3)}^{\text {endo }}\right)$ to $-17.54 \mathrm{G}$ (see Table 8 in ref. 74, QRHF reference wave function). Similar poor performance compared to experiment is also observed for other ab initio methods such as various configuration interaction or perturbation theory based methods. ${ }^{70,72}$ Thus, DFT methods, in particular when combined with the
B3LYP functional, provide a more accurate description of isotropic HFCCs in the allyl radical compared to contemporary electron-correlated $a b$ initio methods, since the latter appear incapable of providing a balanced description of spin polarization. So far, highly correlated ab initio methods beyond the CCSD model have not been applied to study the allyl radical due to the size of this molecule.

Before we turn to the discussion of ZPVCs to isotropic hydrogen HFCCs, we briefly consider the mechanism responsible for the non-vanishing isotropic HFCCs in the allyl radical. Since this radical has a ${ }^{2} A_{2}$ ground state with an unpaired electron residing in the SOMO of $a_{2}$ symmetry (see Fig. 2), the direct spindensity contribution (first term in eqn (1)) to the isotropic HFCCs of hydrogens vanishes. Therefore, as we already mentioned, the $\pi, \pi$-type spin polarization effects are responsible for the non-zero isotropic HFCCs if ZPVCs are neglected. In the DFT-RU approach, this fact manifests itself in that the only nonvanishing elements of the Lagrangian vector (the second term in eqn (1)) are those associated with triplet orbital rotations (TORs) between $\pi$-type molecular orbitals. Furthermore, a detailed analysis of the Lagrangian vector reveals that the dominant spin-polarization contribution is associated with TORs involving the HOMO and LUMO (see Fig. 2), and only a small fraction of spin polarization originates from TORs involving the SOMO. Thus, spin polarization is associated predominantly with $\pi$-type orbitals of $b_{1}$ symmetry, see the pictures of the HOMO and LUMO in Fig. 2. Due to the structure of these orbitals, the isotropic HFCCs of the hydrogens follow a distinct pattern: $A_{\text {eql }}^{\text {iso }}\left(\mathrm{H}_{(2)}\right)$ is positive, $A_{\text {eql }}^{\text {iso }}\left(\mathrm{H}_{(1,3)}^{\text {exo }}\right)$ and $A_{\text {eql }}^{\text {iso }}\left(\mathrm{H}_{(1,3)}\right.$ endo $)$ are negative, and $\left|A_{\text {eql }}^{\text {iso }}\left(\mathrm{H}_{(1,3)}^{\text {exo }}\right)\right|>\left|A_{\text {eql }}^{\text {iso }}\left(\mathrm{H}_{(1,3)}^{\text {endo }}\right)\right|>\left|A_{\text {eql }}^{\text {iso }}\left(\mathrm{H}_{(2)}\right)\right|$. This analysis of the relation between the electronic structure and the isotropic hydrogen HFCCs not only explains the hyperfine structure of the EPR spectrum, but also provides a clue for identifying the vibrational modes that will be important in the computation of the harmonic part of the ZPVCs to the isotropic HFCCs.

Anharmonic and harmonic zero-point vibration corrections, $A_{\mathrm{anh}}^{\text {iso }}$ and $A_{\mathrm{har}}^{\text {iso }}$, to the isotropic HFCCs of hydrogens in the allyl radical are tabulated in Table 1 . We here see that $\left|A_{\mathrm{anh}}^{\text {iso }}\right|$ is negligible for all hydrogens and does not exceed \pm 0.1 G. On the other hand, $A_{\text {har }}^{\text {iso }}$ is negligible only for hydrogens in the terminal $-\mathrm{CH}_{2}$ group, being less than 0.2 $\mathrm{G}$, but it is relatively large for the central hydrogen where $A_{\mathrm{har}}^{\text {iso }}$ becomes $1.24 \mathrm{G}$. Thus, the overall changes in $A_{\mathrm{eql}}^{\text {iso }}\left(\mathrm{H}_{(1,3)}^{\mathrm{exo}}\right)$ and $A_{\text {eql }}^{\text {iso }}\left(\mathrm{H}_{(1,3)}{ }^{\text {endo }}\right)$ due to ZPVCs are small, and only $A_{\text {eql }}^{\text {iso }}\left(\mathrm{H}_{(2)}\right)$ is significantly affected by the ZPVCs (increasing by $29.5 \%$; see $A_{\text {vib }}^{\text {iso }}\left(\mathrm{H}_{(2)}\right)$, in Table 1). The ZPVCs for the central hydrogen is positive $(1.32 \mathrm{G})$ and adding it to $A_{\text {eql }}^{\text {iso }}\left(\mathrm{H}_{(2)}\right)$ does therefore not improve agreement with experiment, the deviation between the computed and the experimental isotropic HFCC values rather increases from $0.41 \mathrm{G}$ to $1.73 \mathrm{G}$. This result indicates that DFT at the B3LYP level overestimates spin polarization effects on the central hydrogen atom and that an account of ZPVCs exposes this deficiency of the B3LYP functional, thus emphasizing the inherent danger of neglecting ZPVCs to isotropic HFCCs in general. Similar observations has been made in connection to exchange-correlation functionals optimized for reproducing experimental magnetic properties. ${ }^{75}$

Let us now analyze the dominant harmonic contribution to the ZPVCs for the central hydrogen in the allyl radical. For 
Table 1 Isotropic hyperfine coupling constants of hydrogens and the zero-point vibrational corrections to these constants in the five allylic radicals studied in this work, computed using the DFT-RU approach ${ }^{a, b}$

\begin{tabular}{|c|c|c|c|c|c|c|c|c|}
\hline \multirow[b]{2}{*}{ Radical } & \multicolumn{5}{|c|}{ Isotropic HFCCs } & \multicolumn{3}{|c|}{ Vibrational corrections } \\
\hline & Atom & $\mathrm{A}_{\mathrm{eql}}^{\mathrm{iso} c}$ & $\mathrm{~A}_{\mathrm{vib}}^{\mathrm{iso} d}$ & $\mathrm{~A}_{\exp }^{\mathrm{iso} e}$ & $\operatorname{Ref}^{f}$ & $\mathrm{~A}_{\mathrm{anh}}^{\mathrm{iso} g}$ & $\mathrm{~A}_{\mathrm{har}}^{\mathrm{iss} h}$ & $\mathrm{~A}_{\mathrm{zpvc}}^{\mathrm{iso}}, \%$ \\
\hline \multirow[t]{3}{*}{ Allyl } & $\mathrm{H}_{(2)}$ & 4.47 & 5.79 & 4.06 & \multirow[t]{3}{*}{69} & 0.08 & 1.24 & 29.53 \\
\hline & $\mathrm{H}_{(1,3)}^{\mathrm{exo}}$ & -15.47 & -15.33 & -14.83 & & -0.04 & 0.18 & 0.90 \\
\hline & $\mathrm{H}_{(1,3)}$ endo & -14.53 & -14.52 & -13.93 & & -0.06 & 0.07 & 0.07 \\
\hline \multirow[t]{3}{*}{ Cyclobutenyl } & $\mathrm{H}_{(2)}$ & 3.81 & 4.04 & 2.41 & \multirow[t]{3}{*}{76} & 0.07 & 0.16 & 6.04 \\
\hline & $\mathrm{H}_{(1,3)}$ & -16.60 & -15.52 & -15.20 & & -0.01 & 1.09 & 6.51 \\
\hline & $\mathrm{H}_{(\beta)}$ & 5.96 & 6.98 & 4.45 & & 0.08 & 0.94 & 17.11 \\
\hline \multirow[t]{3}{*}{ Cyclopentenyl } & $\mathrm{H}_{(2)}$ & 3.91 & 4.29 & 2.77 & \multirow[t]{3}{*}{77} & 0.06 & 0.32 & 9.72 \\
\hline & $\mathrm{H}_{(1,3)}$ & -15.32 & -14.77 & 14.30 & & -0.04 & 0.55 & 3.34 \\
\hline & $\mathrm{H}_{(\beta)}$ & 25.37 & 26.35 & 21.50 & & -0.22 & 1.20 & 3.86 \\
\hline \multirow[t]{5}{*}{ Bicyclo[3.1.0]hexenyl } & $\mathrm{H}_{(2)}$ & 3.67 & 3.97 & 2.54 & \multirow[t]{5}{*}{78} & 0.06 & 0.24 & 8.17 \\
\hline & $\mathrm{H}_{(1,3)}$ & -14.34 & -13.95 & -13.66 & & -0.02 & 0.41 & 2.72 \\
\hline & $\mathrm{H}_{(\beta)}$ & 13.84 & 14.47 & 12.60 & & -0.14 & 0.63 & 3.51 \\
\hline & $\mathrm{H}_{(\delta)}$ & -3.74 & -3.76 & -3.75 & & 0.01 & -0.03 & 0.53 \\
\hline & $\mathrm{H}_{\left(\delta^{\prime}\right)}$ & -3.78 & -3.81 & -3.55 & & -0.02 & -0.01 & 0.79 \\
\hline \multirow[t]{8}{*}{ 1-Hydronaphtyl } & $\mathrm{H}_{(2)}$ & 3.68 & 4.22 & 2.74 & \multirow[t]{8}{*}{79} & 0.06 & 0.48 & 14.67 \\
\hline & $\mathrm{H}_{(1)}$ & -11.77 & -11.86 & -10.70 & & -0.12 & 0.03 & 0.76 \\
\hline & $\mathrm{H}_{(3)}$ & -13.58 & -13.35 & -13.01 & & 0.01 & 0.23 & 1.69 \\
\hline & $\mathrm{H}_{(\beta)}$ & 38.33 & 39.76 & 34.05 & & 0.03 & 1.40 & 3.73 \\
\hline & $\mathrm{H}_{(\mathrm{o})}$ & -3.06 & -3.21 & -2.78 & & -0.01 & -0.14 & 4.90 \\
\hline & $\mathrm{H}_{(\mathrm{m})}$ & 1.39 & 1.55 & 1.00 & & 0.02 & 0.14 & 11.51 \\
\hline & $\mathrm{H}_{(\mathrm{p})}$ & -3.48 & -3.59 & -3.09 & & -0.01 & -0.10 & 3.16 \\
\hline & $\mathrm{H}_{\left(\mathrm{m}^{\prime}\right)}^{(\mathrm{p})}$ & 1.36 & 1.53 & 1.00 & & 0.01 & 0.16 & 12.51 \\
\hline
\end{tabular}

${ }^{a}$ Isotropic HFCCs are given in Gauss. ${ }^{b}$ Isotropic HFCCs evaluated using the B3LYP functional with the HuzIII-su3 basis set. In computations of ZPVCs, the quadratic and cubic force fields have been obtained using the B3LYP functional and the TZV2P basis set. ${ }^{c}$ Isotropic HFCC computed at equilibrium geometry of radical. ${ }^{d}$ Isotropic HFCC with zero-point vibrational corrections added to it's value at equilibrium geometry. ${ }^{e}$ Experimentally determined isotropic HFCC. For conventional EPR experiments sign of isotropic HFCC assigned based on our DFT-RU calculation results. ${ }^{f}$ Reference to experimental work from which $\mathrm{A}_{\exp }$ iso has been taken. ${ }^{g}$ Anharmonic zero-point vibrational correction to isotropic HFCC at effective geometry. ${ }^{h}$ Harmonic zero-point vibrational correction to isotropic HFCC at equilibrium geometry. ${ }^{i}$ Size of zero-point vibrational corrections contribution in $\%$ to total isotropic HFCC, which is computed as $\left|\left(\mathrm{A}_{\text {vib }}^{\text {iso }}-\mathrm{A}_{\text {eql }}^{\text {iso }}\right) / A_{\text {eq }}^{\text {iso } \mid}\right| 100 \%$.
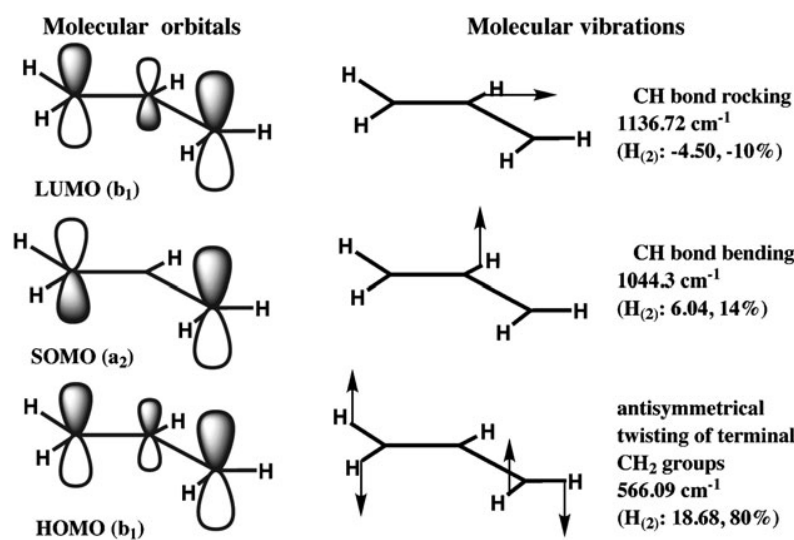
CH bond bending
$1044.3 \mathrm{~cm}^{-1}$ $\left(\mathrm{H}_{(2)}: \mathbf{6 . 0 4}, \mathbf{1 4} \%\right)$

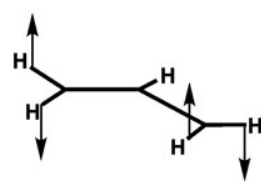

antisymmetrical twisting of terminal$\mathrm{CH}_{2}$ groups $566.09 \mathrm{~cm}^{-1}$

$\left(\mathrm{H}_{(2)}: \mathbf{1 8 . 6 8}, \mathbf{8 0} \%\right)$

Fig. 2 Molecular orbitals of the allyl radical and the main vibrational modes contributing to the harmonic ZPVC to the isotropic HFCC of the central $\mathrm{H}_{(2)}$ hydrogen in this radical. For each depicted vibrational mode we give in parenthesis: the name of hydrogen, magnitude of the partial $\partial^{2} A_{\text {eff }}^{\text {iso }} / \partial Q_{K}^{2}$ derivative in $G / a u^{2}$, and contribution of this mode to the total harmonic ZPVC in percent.

this purpose, we have in Fig. 2 plotted the three vibrational modes that give the largest contributions to $A_{\text {har }}^{\text {iso }}\left(\mathrm{H}_{(2)}\right)$ : (1) the central $\mathrm{CH}$ bond rocking; (2) the central $\mathrm{CH}$ bond bending; and (3) the antisymmetric twisting of the terminal $-\mathrm{CH}_{2}$ groups accompanied with a low amplitude motion of the central hydrogen atom. According to eqn (2), part of the total harmonic ZPVC covered by a single vibrational mode depends on the frequency of the mode and the magnitude of the second derivative of $A_{\mathrm{eff}}^{\text {iso }}$ with respect to the normal coordinates of this vibrational mode. Among the three vibrational modes plotted in Fig. 2 the antisymmetric twisting of the $-\mathrm{CH}_{2}$ groups is responsible for $80 \%$ of $A_{\text {har }}^{\text {iso }}\left(\mathrm{H}_{(2)}\right)$, while the individual contributions from the remaining two vibrations associated with the central $\mathrm{CH}$ group motion are considerably smaller and furthermore effectively cancel each other due to their opposite signs. The main reason for the antisymmetric twisting of the terminal $-\mathrm{CH}_{2}$ groups to play a dominant role in defining the size of $A_{\text {har }}^{\text {iso }}\left(\mathrm{H}_{(2)}\right)$ is that the distortion along the normal coordinate of this vibration introduces a non-vanishing direct spin-density contribution to $A_{\text {eff }}^{\text {iso }}\left(\mathrm{H}_{(2)}\right)$ (see the SOMO localization in Fig. 2) and consequently $\partial^{2} A_{\text {eff }}^{\text {iso }} / \partial Q_{K}^{2}$ is rather large for this mode, $18.68 \mathrm{G} /(\text { a.u. })^{2}$. The two other vibrations plotted in Fig. 2 do not affect the allyl radical SOMO and the distortions along the normal coordinates of these vibrations do therefore not introduce any direct contribution to the spindensity of $A_{\text {eff }}^{\text {iso }}\left(\mathrm{H}_{(2)}\right)$, but rather influence the HOMO and LUMO orbitals which are responsible for the spin-polarization contribution to $A_{\text {eff }}^{\text {iso }}\left(\mathrm{H}_{(2)}\right)$. This second mechanism for generating the non-vanishing $\partial^{2} A_{\text {eff }}^{\text {iso }} / \partial Q_{K}^{2}$, which involves only spin polarization, is less effective than the first one that is dependent on the direct spin-density participation. This fact is reflected by the smaller values of $\partial^{2} A_{\text {eff }}^{\text {iso }} / \partial Q_{K}^{2}$ with respect to vibrations involving the motion of the central $\mathrm{CH}$ bond (see Fig. 2). Thus, we would like to point out that vibrations capable of distorting the molecule in a way that allows for a large direct 
spin-density contribution to the isotropic HFCC of the targeted hydrogen are the most important ones in computations of harmonic ZPVCs to isotropic HFCCs. In the case of the central hydrogen of the allyl radical, which we have analyzed in detail here, $A_{\text {har }}^{\text {iso }}\left(\mathrm{H}_{(2)}\right)$ is dominated by a single vibrational contribution, namely the antisymmetric twisting of the terminal $-\mathrm{CH}_{2}$ groups, and validate in this case the notion that the ZPVC to the isotropic HFCCs is in essence an effect dependent on a single vibrational mode.

\section{B. Cyclobutenyl radical}

Studies of the cyclobutenyl radical are considerably fewer in number than for its parent compound, the allyl radical, and to the best of our knowledge only experimental investigations of the hyperfine structure of its EPR spectra have been carried out until now, see Krusic et al. ${ }^{76}$. The lack of theoretical studies of the isotropic HFCCs of the cyclobutenyl radical might be explained by the fact that its electronic structure is perceived to be very similar to the allyl radical; this radical has also a ${ }^{2} A_{2}$ ground state and its isotropic HFCCs for the hydrogens, including the $\beta$ hydrogens (see Fig. 1), are, in analogy to the allyl radical, determined solely by spin polarization effects. Despite these similarities, the isotropic HFCCs of the hydrogens in the cyclobutenyl radical computed at the equilibrium geometry (see Table 1) do not agree so well with experiment, showing discrepancies of $\pm 1.5 \mathrm{G}$ compared to $\pm 1.0 \mathrm{G}$ for the allyl radical. This result indicates that the overestimation of spin polarization by the B3LYP functional is more pronounced in the cyclobutenyl radical than for its parent compound. Despite this shortcoming, our DFT-RU calculations recover correctly the typical alternation pattern of the isotropic HFCCs of the hydrogens located on the allylic fragment and provides overall a semiquantitative level of accuracy.

As one can see from Table 1, in analogy to the allyl radical, the anharmonic ZPVCs to $A_{\text {eql }}^{\text {iso }}$ is negligible for all hydrogens in the cyclobutenyl radical and their absolute values do not exceed $0.1 \mathrm{G}$. The harmonic ZPVC to $A_{\text {eff }}^{\text {iso }}$, on the other hand, is rather small only for the central hydrogen of the allylic fragment of this radical and for all remaining hydrogens $A_{\text {har }}^{\text {iso }}$ is positive, around 1G. Thus, the harmonic ZPVCs is completely different for the cyclobutenyl radical compared to the allyl radical, in which only the central hydrogen exhibited significant harmonic ZPVC to its isotropic HFCCs. The main reason for the small $A_{\mathrm{har}}^{\text {iso }}\left(\mathrm{H}_{(2)}\right)$ in the cyclobutenyl radical is the unfavorable structure of its vibrational subsystem, as none of the vibrational modes induces a distortion of effective geometry that would allow for a significant direct spin-density contribution to $A_{\text {eff }}^{\text {iso }}\left(\mathrm{H}_{(2)}\right)$. This finding reaffirms the observation made above that the spin polarization is rather ineffective as a mechanism for generating large $\partial^{2} A_{\text {eff }}^{\text {iso }} / \partial Q_{K}^{2}$ derivatives, required for obtaining a non-negligible harmonic ZPVC to $A_{\text {eff }}^{\text {iso }}$. The other two hydrogens, $\mathrm{H}_{(1)}$ and $\mathrm{H}_{(3)}$, associated with the allylic fragment of the cyclobutenyl radical feature rather significant $A_{\text {har }}^{\text {iso }}$, with a value of $1.1 \mathrm{G}$. As one can see from Fig. 3, the harmonic ZPVC contribution to $A_{\text {har }}^{\text {iso }}\left(\mathrm{H}_{(1,3)}\right)$ arises from a single vibration, namely the antisymmetric bending of the terminal $\mathrm{CH}$ bonds in the allylic

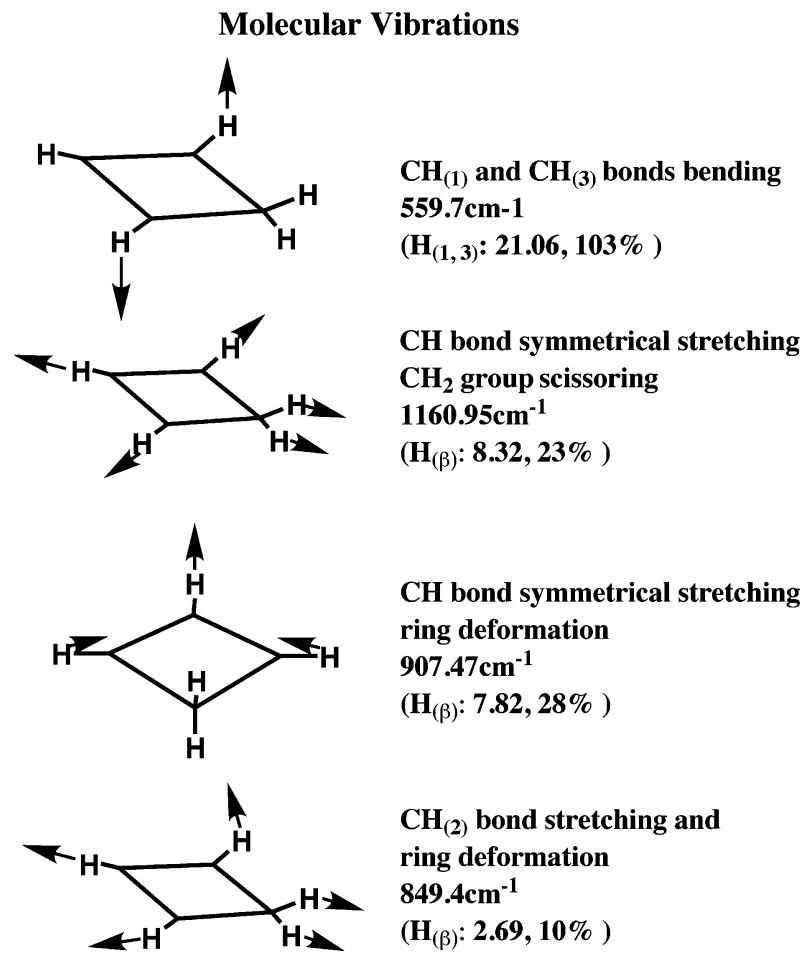

Fig. 3 Main vibrational modes contributing to the harmonic ZPVCs to the isotropic HFCCs of the $\mathrm{H}_{(1,3)}$ and $\mathrm{H}_{(\beta)}$ hydrogens in the cyclobutenyl radical. For each depicted vibrational mode we give in parenthesis: the name of hydrogen, magnitude of the partial $\partial^{2} A_{\text {eff }}^{\text {iso }} / \partial Q_{K}^{2}$ derivative in $G / a u^{2}$, and contribution of this mode to the total harmonic ZPVC in percent.

fragment, as this vibrational mode allows for a strong direct spin-density contribution from the SOMO of $a_{2}$ symmetry to $A_{\text {eff }}^{\text {iso }}\left(\mathrm{H}_{(1,3)}\right)$. The other two hydrogens which exhibit large $A_{\text {har }}^{\text {iso }}$ are the two equivalent $\beta$ hydrogens for which $A_{\text {har }}^{\text {iso }}$ is $0.94 \mathrm{G}$. The $\beta$ hydrogens stand out from the remaining hydrogens as they are not lying in the plane of the allylic fragment and only because they are positioned exactly on the principal symmetry axis, the direct spin-density contribution to their isotropic HFCCs is zero. This special feature of $\mathrm{H}_{\beta}$ influences the harmonic ZPVC to its $A_{\text {eff }}^{\text {iso }}$ significantly. In terms of the magnitude of $A_{\mathrm{eff}}^{\text {iso }}\left(\mathrm{H}_{(\beta)}\right)$, it is only slightly different from $A_{\text {eff }}^{\text {iso }}\left(\mathrm{H}_{(1,3)}\right)$, but the unique position of the $\beta$ hydrogens with respect to the SOMO of the cyclobutenyl radical (see Fig. 3) makes $A_{\mathrm{eff}}^{\text {iso }}\left(\mathrm{H}_{(\beta)}\right)$ dominant due to contributions from three vibrational modes. These vibrations are depicted in Fig. 3. They share a common feature, namely that distortions along the normal coordinates for each of the vibrations lead to a non-vanishing direct spin-density contribution to $A_{\mathrm{eff}}^{\mathrm{iso}}\left(\mathrm{H}_{(\beta)}\right)$ from the SOMO of the cyclobutenyl radical. As shown in Fig. 3, these three vibrational modes contribute up to $61 \%$ of the total $A_{\mathrm{har}}^{\text {iso }}\left(\mathrm{H}_{(\beta)}\right)$, while the remaining part originates in small contributions associated with the other vibrational modes (not depicted in Fig. 3). Thus, the harmonic ZPVCs of the $\beta$ hydrogens in this radical is due to vibrational multimode effects and can consequently not be rationalized using a single vibrational mode model. This finding clearly shows that in some cases such as the $\beta$ hydrogens described above, the only feasible way of evaluating the harmonic part of the 
ZPVCs is by full-scale calculations of this correction with all vibrational modes accounted for. Let us conclude the discussion of the cyclobutenyl radical by establishing the impact of these ZPVCs. The addition of ZPVCs to $A_{\text {eql }}^{\text {iso }}$ improves the agreement between computed and experimental isotropic HFCCs for the terminal hydrogens of the allyl fragment; upon addition of ZPVCs, $A_{\mathrm{eff}}^{\text {iso }}\left(\mathrm{H}_{(1,3)}\right)$ increases from $-16.60 \mathrm{G}$ to $-15.52 \mathrm{G}$ (see $A_{\mathrm{eff}}^{\text {iso }}$ and $A_{\text {eff }}^{\text {iso }}$ in Table 1 ) and consequently becomes in better agreement with the experimental result, which is $-15.20 \mathrm{G}$. For the remaining hydrogens of the cyclobutenyl radical, the correction of $A_{\text {eql }}^{\text {iso }}$ with ZPVCs leads to larger deviations of from the experimental data, with the $\beta$ hydrogens being most affected. Accounting for vibrational corrections in calculations of isotropic HFCCs of hydrogens leads for both the allyl and cyclobutenyl radicals to a similar effect, but the importance of the changes in the HFCCs for the specific hydrogens varies due to the different size of the ZPVCs in the two radicals. As a final note on the cyclobutenyl radical, we note that the isotropic HFCCs of the $\beta$ hydrogens in this radical behaves in a similar fashion as the isotropic HFCC of the central hydrogen of the allylic fragment, but the origin of the harmonic zero-point vibration correction is different for these hydrogens in the two radicals.

\section{Cyclopentenyl radical}

The third radical studied in this work has, as was the case for the cyclobutenyl radical, only been studied experimentally. ${ }^{77}$ The main difference between cyclopentenyl and cyclobutenyl is the number of $\beta$ hydrogens and their location with respect to the principal axis (see Fig. 1): in cyclobutenyl the two equivalent $\beta$ hydrogens are located on the principal axis while in cyclopentenyl four equivalent $\beta$ hydrogens are displaced away from the principal symmetry axis. This positioning of the $\beta$ hydrogens in the latter radical leads to a non-vanishing direct spin-density contribution to the isotropic HFCCs from the SOMO of $a_{2}$ symmetry and makes these constants the largest among the isotropic HFCCs of the cyclopentenyl radical. More specifically, at the equilibrium geometry, the direct spin-density contribution to $A_{\mathrm{eq}}^{\mathrm{iso}}\left(\mathrm{H}_{(\beta)}\right)$ is $17.36 \mathrm{G}$ and the spin polarization contribution to $A_{\mathrm{eq} 1}^{\text {iso }}\left(\mathrm{H}_{(\beta)}\right)$ is $8.01 \mathrm{G}$. This result again emphasizes the importance of the direct spindensity contribution mechanism for obtaining large isotropic HFCCs. The $A_{\text {eql }}^{\text {iso }}$ of the remaining hydrogens in cyclopentenyl behaves similarly to the previous two allylic radicals and arise solely from spin polarization effects. Comparing the DFT-RU results with experimental data, we can from Table 1 see that the computed $A_{\text {eql }}^{\text {iso }}$ of the hydrogens located on the allylic fragment follow the trend observed for the allyl and cyclobutenyl radicals, and on average the deviations from the experimental results do not exceed \pm 1.2 G. Unfortunately, the $\beta$ hydrogens show worse agreement with experiment and the computed $A_{\mathrm{eql}}^{\text {iso }}\left(\mathrm{H}_{(\beta)}\right)$ overshoot the experimental value by almost $4 \mathrm{G}$. The reason for this large error is probably due to the interplay of two effects: the overestimation of the spin polarization contribution to $A_{\mathrm{eq} 1}^{\mathrm{iso}}\left(\mathrm{H}_{(\beta)}\right)$ and a shortcoming of the B3LYP functional to accurately describe the delocalization of the SOMO, which is responsible for the direct spindensity contribution to $A_{\mathrm{eq}}^{\text {iso }}\left(\mathrm{H}_{(\beta)}\right)$. Further investigations appear necessary in order to understand these observations and to design a computational solution that may provide a uniform accuracy for the isotropic hydrogen HFCCs in radicals such as cyclopentenyl, without deteriorating the accuracy obtained for the other hydrogen HFCCs.

The anharmonic and harmonic ZPVCs to the isotropic hydrogen HFCCs in the cyclopentenyl radical is similar to the two smaller allylic radicals described above. Thus, the anharmonic ZPVC is negligible for all hydrogens located on the allylic fragment, while the harmonic ZPVC is rather large as it reaches up to around $0.5 \mathrm{G}$; that is $0.32 \mathrm{G}$ for the central hydrogen and $0.55 \mathrm{G}$ for the terminal hydrogens, respectively. Both $A_{\text {har }}^{\text {iso }}\left(\mathrm{H}_{(2)}\right)$ and $A_{\text {har }}^{\text {iso }}\left(\mathrm{H}_{(1,3)}\right)$ arise mainly from a single vibrational mode, namely the antisymmetric terminal $\mathrm{CH}$ bond rocking which is similar to the vibrational mode depicted in Fig. 3 for the cyclobutenyl radical. For the $\beta$ hydrogens, the anharmonic and harmonic ZPVCs are both non-negligible; $A_{\mathrm{anh}}^{\text {iso }}\left(\mathrm{H}_{(\beta)}\right)$ is $-0.22 \mathrm{G}$ and $A_{\text {har }}^{\text {iso }}\left(\mathrm{H}_{(\beta)}\right)$ is $1.20 \mathrm{G}$, respectively. Thus, the $\beta$ hydrogens of the cyclopentenyl radical are examples where the anharmonicity of the force field at the equilibrium geometry provides an efficient pathway for obtaining a sizable contribution to the total ZPVCs for the hydrogen HFCCs. $A_{\text {har }}^{\text {iso }}\left(\mathrm{H}_{(\beta)}\right)$ also exhibits a novel behavior compared to the previously considered harmonic ZPVCs, that is, it has a true multimode nature and at least 8 vibrational modes, depicted in Fig. 4, contribute to $A_{\mathrm{har}}^{\mathrm{iso}}\left(\mathrm{H}_{(\beta)}\right)$. This behavior of $A_{\text {har }}^{\text {iso }}\left(\mathrm{H}_{(\beta)}\right)$ can be readily understood recalling the fact that both the direct spin-density and the spin polarization contributions to $A_{\text {eff }}^{\text {iso }}\left(\mathrm{H}_{(\beta)}\right)$ are non-vanishing, and consequently that there are many vibrational modes which have non-negligible $\partial^{2} A_{\text {eff }}^{\text {iso }} / \partial Q_{K}^{2}$, contributing to $A_{\text {har }}^{\text {iso }}\left(\mathrm{H}_{(\beta)}\right)$. For the $\beta$ hydrogens, the only way to estimate the ZPVCs is a full-scale computation of both the harmonic and anharmonic ZPVCs, including all vibrational modes of the radical. The local single dominant vibrational mode approach is clearly not applicable in this case. We conclude the discussion of the isotropic HFCCs in the cyclopentenyl radical by noting that, in analogy with the cyclobutenyl radical case, an addition of the ZPVCs to $A_{\mathrm{eql}}^{\text {iso }}$ improves the agreement with experiment for the terminal hydrogens of the allylic fragment, while for the central hydrogen of the allylic fragment as well as for the $\beta$ hydrogens the opposite is true. Therefore, despite the different nature of the ZPVCs for some hydrogens in the cyclopentenyl radical compared to cyclobutenyl and allyl radicals, the general effect of accounting for zero-point vibrational corrections to the isotropic HFCCs lead to a similar impact for all three allylic radicals.

\section{Bicyclo[3.1.0|hexenyl radical}

The bicyclo[3.1.0]hexenyl radical combines the main features of the cyclobutenyl and cyclopentenyl radicals. In addition to the hydrogens associated with the allylic fragment, which are common for all three radicals, it also has two distinct sets of hydrogens with a different nature for their isotropic HFCCs. The first set of hydrogens consist of $\mathrm{H}_{(\delta)}$ and $\mathrm{H}_{\left(\delta^{\prime}\right)}$, which are located in the symmetry plane of the molecule and consequently their isotropic HFCCs are determined solely by spin-polarization effects as was the case for the $\beta$ hydrogens 

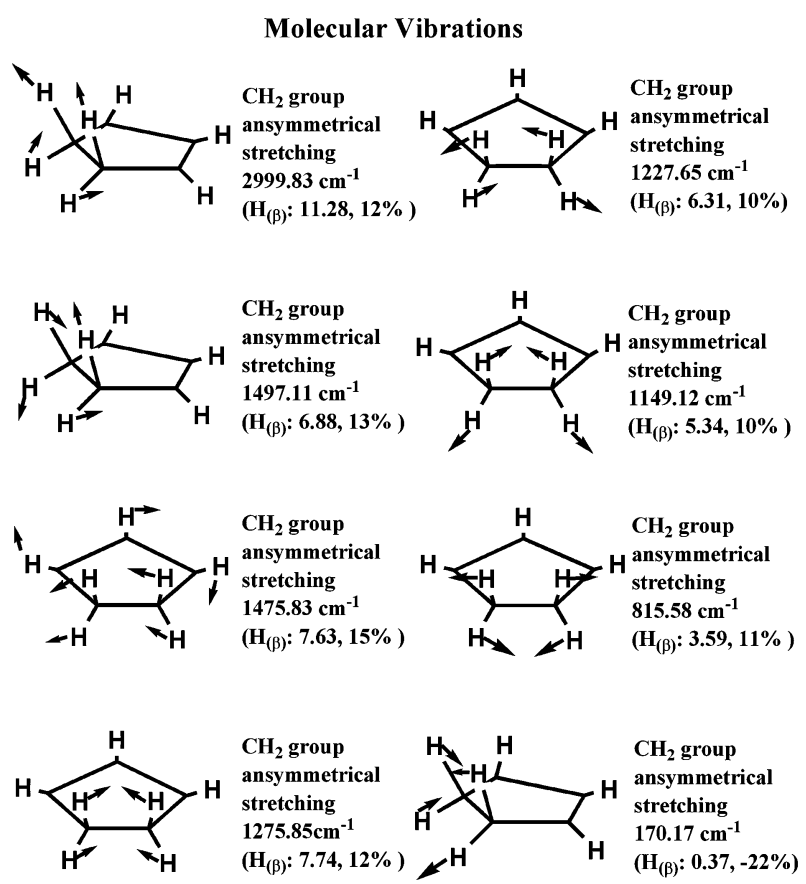

Fig. 4 Main vibrational modes contributing to the harmonic ZPVC to the isotropic HFCC of the $\mathrm{H}_{(\beta)}$ hydrogens in the cyclopentenyl radical. For each depicted vibrational mode we give in parenthesis: the name of hydrogen, magnitude of the partial $\partial^{2} A_{\text {eff }}^{\text {iso }} / \partial Q_{K}^{2}$ derivative in $G / a u^{2}$, and contribution of this mode to the total harmonic ZPVC in percent.

in the cyclobutenyl radical. The second set of hydrogens consists of two equivalent $\beta$ hydrogens that are positioned out of the symmetry plane of the molecule, and consequently spin polarization gives a significant direct contribution to these isotropic HFCCs, as was the case for the cyclopentenyl radical. Due to these similarities with the cyclobutenyl and cyclopentenyl radicals, the isotropic hydrogen HFCCs of the bicyclo[3.1.0]hexenyl radical also behaves in a similar fashion, i.e. the isotropic HFCCs computed at the equilibrium geometry overestimate, in terms of absolute values, the experimental results ${ }^{78}$ for all hydrogens except $\mathrm{H}_{(\delta)}$ for which $A_{\text {eff }}^{\text {iso }}$ almost exactly matches the EPR measurement. We note that $\mathrm{H}_{\left(\delta^{\prime}\right)}$ has a much smaller overestimation of the HFCC than any of the other hydrogens in this radical. This indicates that the spin polarization contribution, which exclusively defines the isotropic HFCCs of these hydrogens, becomes less sensitive to the quality of the orbital description responsible for the spin polarization with increasing distance between these orbitals and the hydrogen for which the isotropic HFCC is computed. To illustrate this finding, let us consider $A_{\text {eql }}^{\text {iso }}$ for the hydrogens of the allylic fragment of the bicyclo[3.1.0]hexenyl radical and the two $\delta$ hydrogens. An analysis of the Lagrangian vector indicates that the TOR involving the HOMO and LUMO orbitals is responsible for the major part of the spin polarization effect. Thus, since the HOMO and LUMO orbitals are located on three carbons of the allylic fragment, we expect a larger error in the spin polarization contribution to $\left|A_{\text {eq }}^{\text {iso }}\right|$ for hydrogens of the allylic fragment compared to the $\delta$ hydrogens. Indeed, according to the results presented in Table 1 , the computed $\left|A_{\text {eql }}^{\text {iso }}\right|$ values deviate from the experimental ones by roughly $1 \mathrm{G}$ for the former set of hydrogens and only at most by $0.33 \mathrm{G}$ for the latter set of hydrogens. This finding is important from a practical point of view, as it indicates that the shortcomings of the B3LYP functional in providing a balanced description of the $\pi, \pi$ spin polarization has a local nature and is most pronounced in the vicinity of the HOMO and LUMO orbitals. Consequently, the isotropic HFCCs of hydrogens far away from the allylic fragment on which the HOMO and LUMO orbitals are located can be described with greater accuracy. However, it is yet hard to judge if this trend is genuine for all larger allylic radicals or if it is specific to bicyclo[3.1.0]hexenyl.

The anharmonic and harmonic ZPVCs to the isotropic hydrogen HFCCs of the bicyclo[3.1.0]hexenyl radical can be separated into two groups by the nature of these corrections, where the first group comprises the ZPVCs which are located on the allylic fragment of the bicyclo[3.1.0]hexenyl radical as well as the $\beta$ hydrogens, and where the second group comprises ZPVCs for the $\delta$ hydrogens. The ZPVCs of the first group behaves similarly to the corresponding ZPVCs for the cyclopentenyl radical, which have been described in detail above, and the same mechanism is responsible for the harmonic part of the ZPVCs due to the structural and electronic similarity of these two radicals. Furthermore, the harmonic ZPVCs dominated by a single vibrational mode, like the ones encountered for the hydrogens of the radical allylic fragments, are very close in size for the two radicals (see Table 1): $A_{\text {har }}^{\text {iso }}\left(\mathrm{H}_{(2)}\right)$ is $0.32 \mathrm{G}$ in cyclopentenyl and $A_{\text {har }}^{\text {iso }}\left(\mathrm{H}_{(2)}\right)$ in $0.24 \mathrm{G}$ in bicyclo[3.1.0]hexenyl; $A_{\text {har }}^{\text {iso }}\left(\mathrm{H}_{(1,3)}\right)$ is $0.55 \mathrm{G}$ in cyclopentenyl and $A_{\text {har }}^{\text {iso }}\left(\mathrm{H}_{(1,3)}\right)$ is $0.41 \mathrm{G}$ in bicyclo[3.1.0]hexenyl. Thus, ZPVCs of this kind seem to be transferable between electronically and structurally similar radicals and, consequently, it seems sufficient to compute ZPVCs for the parent radical in order to approximately estimate these corrections in its derivatives. Unfortunately, this trend does not hold for the $\beta$ hydrogens for which the harmonic ZPVCs differ between the two radicals by a factor of two, $A_{\text {har }}^{\text {iso }}\left(\mathrm{H}_{(\beta)}\right)$ is $1.20 \mathrm{G}$ for the cyclopentenyl and $0.63 \mathrm{G}$ for bicyclo[3.1.0]hexenyl, due to the multimode nature of the harmonic ZPVC of the $\beta$ hydrogens, leading to a nonlocal dependence on the structure of the radical.

The ZPVCs from the second group, which consist of two $\delta$ hydrogens, behave differently from the first group with both anharmonic and harmonic ZPVCs being negligible. The small magnitude of the anharmonic ZPVCs is in line with our finding for other isotropic HFCCs of hydrogens defined solely by spin polarization effects, such as the hydrogens located on the allylic fragment of the radical. However, the small magnitude of the harmonic ZPVCs for the $\delta$ hydrogens calls for a closer examination: A detailed analysis of $A_{\text {har }}^{\text {iso }}$ for these hydrogens shows that for all vibrational modes, $\partial^{2} A_{\text {eff }}^{\text {iso }} / \partial Q_{K}^{2}$ is small due to the fact that the direct spin-density contribution to $A_{\text {eff }}^{\text {iso }}$ is negligible in all cases when distorting along the normal coordinates of the vibrational mode. The main reason for the significantly diminished efficiency of the direct spindensity mechanism for the generation of sizable $\partial^{2} A_{\text {eff }}^{\text {iso }} / \partial Q_{K}^{2}$ derivatives is the large separation between the SOMO on which the unpaired electron is located and the hydrogen of interest. This in a turn leads to a small spin density at the 
position of this hydrogen. Taking this into account, we conclude that the ZPVCs to the isotropic hydrogen HFCCs, which are dominated by spin polarization effects, becomes rather small in cases when the separation between the hydrogen and the SOMO orbital is large in $\pi$-type radicals. Thus, knowledge of the localization and shape of the SOMO orbital allows for an a priori identification of hydrogen atoms for which significant ZPVCs contributions.

\section{E. 1-Hydronaphthyl radical}

The last allylic radical considered in this work is the 1-hydronaphthyl radical, which, in contrast to the four radicals described previously, has an extended $\pi$-orbital system localized on a phenyl ring and the allylic fragment of this radical, and consequently the mechanism of spin polarization is more complex in this radical. In its ${ }^{2} A^{\prime \prime}$ ground state, an unpaired electron is residing in the SOMO (see Fig. 5) localized predominantly on the allylic fragment and the isotropic HFCCs of all hydrogens lying in the symmetry plane of the molecule are therefore determined solely by the spin polarization effects. However, in the 1-hydronaphthyl radical, the $\pi, \pi$-spin polarization mechanism is more complex and is not limited to the participation of the HOMO and LUMO orbitals as in the allylic radicals discussed above. In fact, a detailed analysis of the Lagrangian vector reveals that it contains three non-vanishing elements, being approximately of the same size, which are associated with the TORs involving the following orbital pairs: HOMO and LUMO, HOMO- 1 and LUMO +1 , and HOMO-2 and LUMO + 2, respectively. All these orbitals are depicted in Fig. 5 and as one can see they are delocalized over the full backbone of the 1-hydronaphthyl radical and therefore provide an efficient pathway for obtaining nonvanishing isotropic HFCCs for the hydrogens localized on both the phenyl ring and the allylic fragment of the radical. In addition to the hydrogens lying in the symmetry plane of the 1-hydronaphthyl radical, there are also two equivalent $\beta$ hydrogens which are located outside the symmetry plane. Consequently, their isotropic HFCCs are to a larger extent determined by the direct spin-density contribution (from the SOMO orbital of $a^{\prime \prime}$ symmetry, see Fig. 5), with the spin polarization contribution being of minor importance in this case.

Let us now compare the hydrogen HFCCs in the 1-hydronaphthyl radical computed using the DFT-RU approach at the equilibrium geometry with the experimental ENDOR data of Böhme and Wolf. ${ }^{79}$ As expected, the computed $A_{\text {eql }}^{\text {iso }}$ of the hydrogens in the 1-hydronaphthyl radical follow the trends observed previously for the smaller allylic radicals: $A_{\text {eql }}^{\text {iso }}$ of hydrogens located on the allylic fragment, in terms of absolute values, overestimate the experimental data by roughly $1 \mathrm{G}$; $A_{\text {eql }}^{\text {iso }}$ of the $\beta$ hydrogens show, similarly to the $\beta$ hydrogens in the cyclopentenyl radical, the largest deviation from the ENDOR results, the difference being $4.28 \mathrm{G} ; A_{\text {eql }}^{\text {iso }}$ of the hydrogens located on the phenyl ring are in the overall best agreement with experimental results with an average deviation of typically less than $0.4 \mathrm{G}$ in magnitude, as was observed for the $\delta$ hydrogens in the bicyclo[3.1.0] radical. These results again indicate that the B3LYP functional is not satisfactory
Molecular orbitals
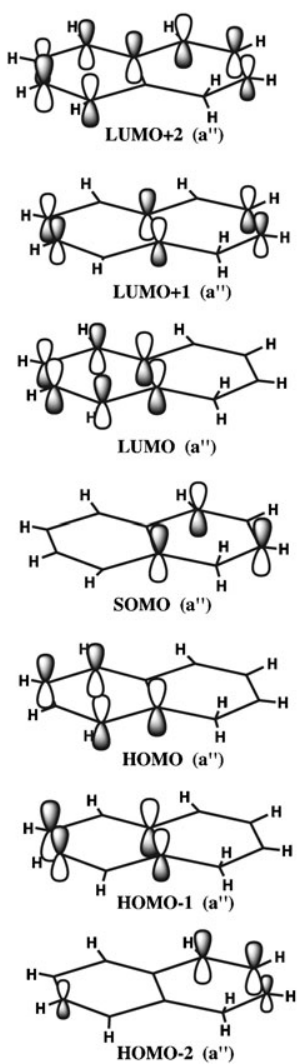

Molecular vibrations
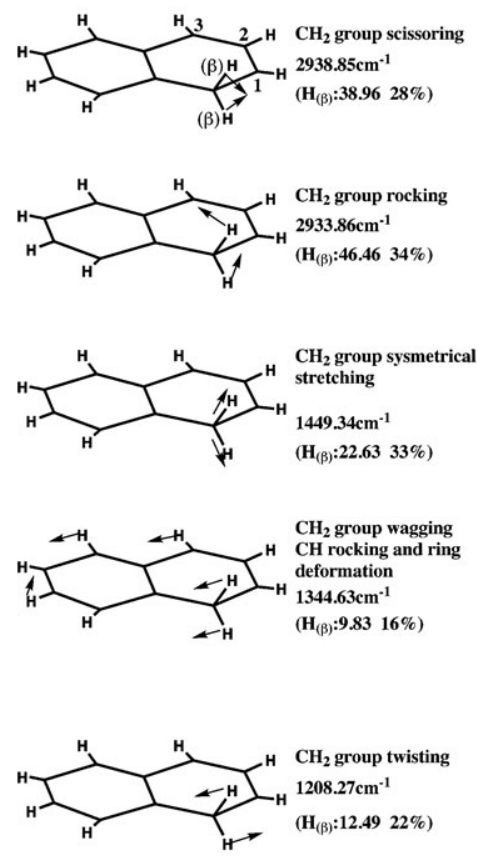

Fig. 5 Molecular orbitals of the 1-hydronaphtyl radical and the main vibrational modes contributing to the harmonic ZPVC to the isotropic HFCC of the central $\mathrm{H}_{(2)}$ hydrogen in this radical. For each depicted vibrational mode we give in parenthesis: the name of hydrogen, magnitude of the partial $\partial^{2} A_{\text {eff }}^{\text {iso }} / \partial Q_{K}^{2}$ derivative in $G / a u^{2}$, and contribution of this mode to the total harmonic ZPVC in percent.

for calculating isotropic HFCCs of hydrogens in allylic radicals as it has a tendency to overestimate, in terms of absolute values, both the direct spin-density and spin polarization contributions to the isotropic HFCCs. This is most likely due to a failure of the functional in capturing the (de)localization of the $\pi$ orbitals, especially the SOMO, on the allylic fragment of these radicals.

Considering the ZPVCs to the HFCCs, we note from Table 1 that the anharmonic and harmonic ZPVCs to the isotropic HFCCs of the hydrogens at the phenyl ring are negligible. This follows from the fact that these hydrogens are far away from the SOMO localized on the allylic fragment and consequently the direct spin-density mechanism for generating sizable $\partial^{2} A_{\text {eff }}^{\text {iso }} \partial Q_{K}^{2}$ derivatives is ineffective, in agreement with our findings for the $\delta$ hydrogens of the bicyclo[3.1.0]hexenyl radical. For the hydrogens of the allylic fragment, with the exception of the terminal hydrogen $\mathrm{H}_{(1)}$, the ZPVCs behave similarly to the corresponding corrections in the allylic hydrogens of the other four radicals investigated in this work-that is, the anharmonic ZPVC to $A_{\text {eql }}^{\text {iso }}$ is negligible and the harmonic ZPVC to $A_{\text {eql }}^{\text {iso }}$ has a singlemode nature with a magnitude being between $0.23 \mathrm{G}$ and 
$0.48 \mathrm{G}$. The only allylic hydrogen which do not fit this trend is $\mathrm{H}_{(1)}$, which exhibits a small but non-negligible anharmonic ZPVC to $A_{\text {eql }}^{\text {iso }}$ and a negligible harmonic

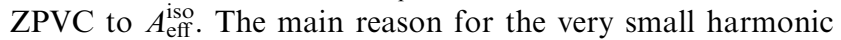
ZPVC to $\mathrm{H}_{(1)}$ in the 1-hydronaphthyl radical is the same as for the terminal hydrogens of the allyl radical, i.e. none of the vibrational modes induces a distortion of the effective geometry of the radical that would allow for a significant direct spin-density contribution to the isotropic HFCCs of this hydrogen.

The two remaining hydrogen HFCCs in the 1-hydronaphthyl radical which we have not yet discussed are the equivalent $\beta$ hydrogens. They exhibit the largest ZPVCs among the hydrogens of all allylic radical investigated in this work. In analogy to the $\beta$ hydrogens of the cyclopentenyl and bicyclo[3.1.0]hexenyl radicals, the harmonic ZPVC to $A_{\mathrm{eff}}^{\mathrm{iso}}\left(\mathrm{H}_{(\beta)}\right)$ has a multimode character; its value is $1.40 \mathrm{G}$, which is slightly larger than the harmonic ZPVC for the $\beta$ hydrogens of the cyclopentenyl radical. Furthermore, due to the smaller anharmonicity of the force field in the region near the $\beta$ hydrogens, the anharmonic ZPVC is negligible and consequently the anharmonic ZPVC does not counteract the harmonic ZPVCs, as is the case for the cyclopentenyl and bicyclo[3.1.0]hexenyl radicals, and this is the reason the total ZPVCs to the isotropic HFCCs of the $\beta$ hydrogens in 1-hydronaphthyl radical is the largest one of our study.

To conclude the discussion of the ZPVCs to the isotropic hydrogen HFCCs of the 1-hydronaphthyl radical, let us examine the effect of these corrections for the agreement between computed and measured isotropic HFCCs. As expected, on the basis of our findings for the smaller allylic radicals considered in this work, only the $\mathrm{H}_{(3)}$ of allylic ring benefits from taking ZPVCs into account in our DFT-RU calculations, for which the deviation between the computed and experimental isotropic HFCC decreases by $0.24 \mathrm{G}$ (see Table 1). For the other hydrogens in the 1-hydronaphthyl radical, the addition of ZPVCs to their isotropic HFCCs computed at the equilibrium geometry leads to a worse agreement with experimental data, and the most pronounced increase of the discrepancies (from $4.28 \mathrm{G}$ to $5.71 \mathrm{G}$, see Table 1) are observed for the $\beta$ hydrogens since they have the largest ZPVCs. Taking all these results into account, we conclude that taking into account the ZPVCs in DFT-RU calculations of isotropic hydrogen HFCCs does not improve the agreement with experimental results for the 1-hydronaphthyl radical, and thus highlight the deficiencies of the B3LYP functional in the description of the electronic structure of the allylic radicals.

\section{F. General trends in allylic radicals}

After describing the isotropic HFCCs of hydrogens in these different allylic compounds and the corresponding ZPVCs, we now summarize our findings and give some general estimates of the importance of these corrections for routine computations. The five allylic radicals have in total 22 unique hydrogens, with 19 hydrogens having their isotropic HFCCs determined solely by spin-polarization effects and 3 hydrogens having their isotropic HFCCs determined by both the direct spin-density contribution of the SOMO and spin-polarization effects. Overall, the isotropic HFCCs computed at the equilibrium geometry are in rather good agreement with the experimental data with a mean absolute deviation (MAD) of $1.07 \mathrm{G}$; the largest deviations are observed for the $\beta$ hydrogens in the cyclopentenyl, bicyclo[3.1.0]hexenyl and 1-hydronaphthyl radicals. Adding ZPVCs to the isotropic HFCCs evaluated at he equilibrium geometry, see $A_{\text {vib }}^{\text {iso }}$ in Table 1 , increases the MAD to $1.30 \mathrm{G}$. Taking ZPVCs into account does therefore not lead to a better agreement with experiment. We note from Table 1 that only for 6 terminal hydrogens located on the allylic fragments of the radicals does the inclusion of ZPVCs lead to improved agreement with experimental data, whereas for the other 15 hydrogens, better agreement with experiment is obtained without considering ZPVCs. These results indicate that the B3LYP exchangecorrelation functional systematically overestimates, in terms of absolute values, the spin polarization as well as the direct spin-density contributions to the isotropic HFCCs in the allylic radicals and only by virtue of error cancelation is the isotropic hydrogen HFCCs evaluated at the equilibrium geometry able to provide better agreement with experimental data than the more complete approach presented in this work. The neglect of ZPVCs in calculations of isotropic HFCCs can therefore potentially be dangerous as it can hide deficiencies in the exchange-correlation functional used in DFT calculations as seen for the B3LYP functional in this work. We therefore advocate that the efforts of tailoring basis set for computations of HFCCs as well as benchmark studies of the performance of exchange-correlation functionals should be carried out using effective experimental isotropic HFCCs references, in which all experimental isotropic HFCCs are corrected for by zeropoint vibrational effects. Such an approach has already been recommended in the context of the calculation of indirect spin-spin coupling constants. ${ }^{80}$

From our DFT-RU results presented in Table 1, the hydrogen ZPVC is on average about $0.45 \mathrm{G}$, where the hydrogens located on the allylic fragment as well as the $\beta$ hydrogens exhibit the largest ZPVCs in terms of absolute values. However, the relative importance of the ZPVCs to the isotropic HFCCs varies significantly going from one type of hydrogen to another. For the $\beta$ hydrogens, which exhibit large direct spin-density contributions to their isotropic HFCCs, the addition of ZPVCs computed at the equilibrium geometry changes these constants only by roughly $3.5 \%$, and consequently the ZPVCs can be safely neglected for hydrogens of this kind in the allylic radicals. On the other hand, for the allylic hydrogens, an account of ZPVCs significantly alter the isotropic HFCCs and these constants increase or decrease by up to almost $30 \%$, being in many cases still very small. An account of ZPVCs is thus essential for obtaining trustworthy theoretical results. Taking this into account, we conclude that the ZPVCs can significantly alter the values of the isotropic HFCCs of specific hydrogens, and consequently the development of analytic methods for evaluation of these corrections are desirable, following e.g. the strategy of Thorvaldsen et al., ${ }^{81}$ as the numerical approach applied in this work is computationally too expensive for large radicals with more than $30-40$ atoms. 


\section{Guidelines for identifying large ZPVCS to hydrogen hfecs in $\pi$-type radicals}

Based on the analysis of our data, we now present a set of simple rules for an approximate identification of hydrogens with isotropic HFCCs in $\pi$-type organic radicals that are significantly affected by ZPVCs. The determination of the magnitude of the hydrogen HFCC ZPVCs follow a four-step procedure that requires knowledge of the localization of the $\pi$ orbitals in which the unpaired electron resides, and a knowledge of the vibrational modes of the radical. Our proposed procedure can be summarized as:

(1) Is the absolute value of the isotropic HFCC larger than $20 \mathrm{G}$ ? If yes, then the zero-point vibrational corrections are relatively small for this constant and can be safely neglected. If no, proceed to second step.

(2) Is the absolute value of the isotropic HFCC smaller than $3 \mathrm{G}$ ? If yes, then proceed to third step. If no, then proceed directly to the fourth step.

(3) If the hydrogen of interest is at least $2.5 \AA$ away from the SOMO orbital, then its ZPVCs can be neglected. If this is not the case, proceed to the fourth step.

(4) If the isotropic hyperfine coupling constant of the hydrogen is determined solely by spin polarization effects, then identify the vibrational modes which generate the geometry distortions capable of introducing a large direct spin-density contribution to the isotropic HFCC. If no modes can be identified, the ZPVC is expected to be small and can be neglected, otherwise one needs to perform calculations of ZPVCs to the isotropic HFCCs taking into account the identified vibrational modes. If the isotropic hyperfine coupling constant of the hydrogen is determined by both direct spin-density and spinpolarization contributions, then the ZPVCs to these constants most likely have multimode nature and can be estimated only by means of a full-scale calculations of the ZPVCs.

We believe these guidelines for identifying hydrogens with significant ZPVC contributions to the isotropic HFCCs will allow problematic hydrogens in organic radicals to be easily noted and aid in the analysis of experimentally measured results. Moreover, they can provide an indication as to whether ZPVCs need to be taken into account in a calculation of isotropic HFCCs of hydrogens. These guidelines can also allow us to select more reliable experimental reference data improving the computational protocols needed for calculation of HFCCs in terms of exchange-correlation functionals and basis sets.

\section{Conclusions}

This paper has presented the first systematic study of zero-point vibration effects on isotropic hyperfine coupling constants in small and medium-sized molecules, using allylic radicals as model systems. The analysis indicates that the widely accepted strategy for investigating hyperfine coupling constants of organic radicals in which zero-point vibrational corrections are simply neglected is not entirely satisfactory and leads to additional errors in practical calculations, which can affect the accuracy of the results obtained. The numerical methodology developed in this work for evaluating zero-point vibrational corrections to isotropic hyperfine coupling constants is applicable to small and medium sized radicals with up to 30-40 atoms. Its efficiency has been demonstrated by calculations of zero-point vibrational corrections to hydrogens hyperfine coupling constants in the 1-hydronaphthyl radical, which probably is the largest of this kind performed until now. However, for even larger radicals, the numerical methodology for evaluating zero-point vibration corrections described in this work becomes computationally too expensive and development of novel analytical approaches capable of evaluating these corrections are therefore desirable. In order to fill this gap and to allow at least for an approximate estimation of zero-point vibrational corrections to isotropic hyperfine coupling constants we have designed, based on the analysis of these corrections in allylic radicals, a set of guidelines for identification of hydrogens which can be expected to exhibit significant zero-point vibrational corrections. We believe these guidelines can serve as a convenient tool for experimentalists and theoreticians alike in their investigations of organic radicals and thus promote a more rigorous understanding of the interplay of different effects in calculations of hyperfine coupling constants of radicals.

\section{Acknowledgements}

KR has been supported by the Research Council of Norway through a CoE grant (Grant No. 179568/V30) and an individual researcher grant (Grant No. 191251). This work has also received support from the Norwegian Supercomputing program through a grant of computer time.

\section{References}

1 E. van Lenthe, P. E. S. Wormer and A. van der Avoird, J. Chem. Phys., 1997, 107, 2488-2498.

2 G. Schreckenbach and T. Ziegler, J. Phys. Chem. A, 1997, 101, 3388-3399.

3 O. L. Malkina, J. Vaara, B. Schimmelpfennig, M. Munzarova, V. G. Malkin and M. Kaupp, J. Am. Chem. Soc., 2000, 122, 9206-9218.

4 F. Neese, J. Chem. Phys., 2001, 115, 11080-11096.

5 K. M. Neyman, D. I. Ganyushin, A. V. Matveev and V. A. Nasluzov, J. Phys. Chem. A, 2002, 106, 5022-5030.

6 Z. Rinkevicius, L. Telyatnyk, P. Salek, O. Vahtras and H. Ågren, J. Chem. Phys., 2003, 119, 10489-10496.

7 I. Malkin, O. L. Malkina, V. G. Malkin and M. Kaupp, J. Chem. Phys., 2005, 123, 244103-16.

8 S. Komorovsky, M. Repisky, O. L. Malkina, V. G. Malkin, I. Malkin and M. Kaupp, J. Chem. Phys., 2006, 124, 084108.

9 Z. Rinkevicius, K. J. de Almeida and O. Vahtras, J. Chem. Phys., 2008, 129, 064109-17.

10 Z. Rinkevicius, K. J. de Almeida, C. I. Oprea, O. Vahtras, H. Ågren and K. Ruud, J. Chem. Theory Comput., 2008, 4, $1810-1828$.

11 V. Barone, C. Adamo and N. Russo, Chem. Phys. Lett., 1993, 212, 5-11.

12 L. A. Eriksson, V. G. Malkin, O. L. Malkina and D. R. Salahub, J. Chem. Phys., 1993, 99, 9756-9763.

13 E. van Lenthe, A. van der Avoird and P. E. S. Wormer, J. Chem. Phys., 1998, 108, 4783-4796.

14 F. Neese, J. Chem. Phys., 2003, 118, 3939-3948.

15 A. V. Arbuznikov, J. Vaara and M. Kaupp, J. Chem. Phys., 2004, 120, 2127-2139.

16 Z. Rinkevicius, L. Telyatnyk, O. Vahtras and H. Ågren, J. Chem. Phys., 2004, 121, 7614-7623. 
17 E. Malkin, I. Malkin, O. L. Malkina, V. G. Malkin and M. Kaupp, Phys. Chem. Chem. Phys., 2006, 8, 4079-4085.

18 C. I. Oprea, L. Telyatnyk, Z. Rinkevicius, O. Vahtras and H. Agren, J. Chem. Phys., 2006, 124, 174103-10.

19 M. R. Pederson and S. N. Khanna, Phys. Rev. B: Condens. Matter Mater. Phys., 1999, 60, 9566.

20 M. Shoji, K. Koizumi, T. Hamamoto, T. Taniguchi, R. Takeda, Y. Kitagawa, T. Kawakami, M. Okumura, S. Yamanaka and K. Yamaguchi, Polyhedron, 2005, 24, 2708-2715.

21 F. Aquino and J. H. Rodriguez, J. Chem. Phys., 2005, 123, 204902-6.

22 R. Takeda, S. Mitsuo, S. Yamanaka and K. Yamaguchi, Polyhedron, 2005, 24, 2238-2241.

23 R. Reviakine, A. V. Arbuznikov, J.-C. Tremblay, C. Remenyi, O. L. Malkina, V. G. Malkin and M. Kaupp, J. Chem. Phys., 2006, 125, 054110-12.

24 S. Sinnecker and F. Neese, J. Phys. Chem. A, 2006, 110, 12267-12275.

25 F. Neese, J. Chem. Phys., 2007, 127, 164112-9.

26 F. Neese, Curr. Opin. Chem. Biol., 2003, 7, 125-135.

27 M. Kaupp, R. Reviakine, O. L. Malkina, A. Arbuznikov, B. Schimmelpfennig and V. G. Malkin, J. Comput. Chem., 2002, 23, 794-803.

28 M. Munzarova and M. Kaupp, J. Phys. Chem. A, 1999, 103, 9966-9983.

29 R. Improta and V. Barone, Chem. Rev., 2004, 104, 1231-1254.

30 M. Perić, B. Engels and S. D. Peyerimhoff, J. Mol. Spectrosc., 1991, 150, 56-59.

31 B. Engels, M. Perić, W. Reuter, S. D. Peyerimhoff and F. Grein, J. Chem. Phys., 1992, 96, 4526-4535.

32 M. Perić and B. Engels, J. Mol. Spectrosc., 1995, 174, 334-352.

33 M. Perić, M. Mladenović and B. Engels, J. Chem. Phys., 2004, 121, 2636-2645.

34 V. Barone, P. Carbonniere and C. Pouchan, J. Chem. Phys., 2005, 122, 224308.

35 C. Puzzarini and V. Barone, J. Chem. Phys., 2008, 129, 084306.

36 C. Puzzarini and V. Barone, Phys. Chem. Chem. Phys., 2008, 10, 6991-6997.

37 C. Puzzarini and V. Barone, J. Chem. Theory Comput., 2009, 5, 2378-2387.

38 V. Barone, J. Bloino and M. Biczysko, Phys. Chem. Chem. Phys., 2010, 12, 1092-1101.

39 M. Pavone, M. Biczysko, N. Rega and V. Barone, J. Phys. Chem. $B, 2010,114,11509-11514$.

40 L. Hermosilla, P. Calle, J. M. Garcia de la Vega and C. Sieiro, J. Phys. Chem. A, 2005, 109, 1114-1124.

41 L. Hermosilla, P. Calle, J. M. Garcia de la Vega and C. Sieiro, J. Phys. Chem. A, 2005, 109, 7626-7635.

42 L. Hermosilla, P. Calle, J. M. García de la Vega and C. Sieiro, J. Phys. Chem. A, 2006, 110, 13600-13608.

43 V. Barone, P. Cimino and E. Stendardo, J. Chem. Theory Comput., 2008, 4, 751-764.

44 C. Adamo, M. Heitzmann, F. Meilleur, N. Rega, G. Scalmani, A. Grand, J. Cadet and V. Barone, J. Am. Chem. Soc., 2001, 123, 7113-7117.

45 N. Rega, M. Cossi and V. Barone, J. Am. Chem. Soc., 1998, 120, $5723-5732$.

46 F. Jolibois, J. Cadet, A. Grand, R. Subra, N. Rega and V. Barone, J. Am. Chem. Soc., 1998, 120, 1864-1871.

47 D. M. Chipman, in Quantum Mechanical Electronic Structure Calculations, Kluwer, Netherlands, 1995, pp. 109-138.

48 J. K. Kochi, P. Bakuzis and P. J. Krusic, J. Am. Chem. Soc., 1973, 95, 1516-1526.
49 S. Deycard, J. Lusztyk, K. U. Ingold, F. Zerbetto, M. Z. Zgierski and W. Siebrand, J. Am. Chem. Soc., 1988, 110, 6721-6726.

50 R. W. Fessenden and R. H. Schuler, J. Chem. Phys., 1965, 43, 2704-2712.

51 R. Livingston and H. Zeldes, J. Chem. Phys., 1966, 44, 1245-1259.

52 Z. Rinkevicius, I. Tunell, P. S. ałek, O. Vahtras and H. A. gren, J. Chem. Phys., 2003, 119, 34-46.

53 C. W. Kern and R. L. Matcha, J. Chem. Phys., 1968, 49, 2081-2091.

54 M. Toyama, T. Oka and Y. Morino, J. Mol. Spectrosc., 1964, 13(1-4), 193-213.

55 K. Ruud, P.-O. Åstrand and P. R. Taylor, J. Chem. Phys., 2000, 112, 2668-2683.

56 P.-O. Åstrand, K. Ruud and P. R. Taylor, J. Chem. Phys., 2000, 112, 2655-2667.

57 P.-O. Åstrand, K. Ruud and D. Sundholm, Theor. Chem. Acc., 2000, 103, 365-373.

58 A. D. Becke, J. Chem. Phys., 1993, 98, 5648-5652.

59 A. D. Becke, Phys. Rev. A: At., Mol., Opt. Phys., 1988, 38, 3098-3100.

60 C. Lee, W. Yang and R. G. Parr, Phys. Rev. B: Condens. Matter, 1988, 37, 785-789.

61 S. H. Vosko, L. Wilk and M. Nusair, Can. J. Phys., 1980, 58, $1200-1211$.

62 A. Schafer, C. Huber and R. Ahlrichs, J. Chem. Phys., 1994, 100, 5829-5835.

63 D. A. Boese, W. Kopper and M. M. L. Jan, Mol. Phys., 2005, 103, 863-876.

64 T. A. Ruden, O. B. Lutnaes, T. Helgaker and K. Ruud, J. Chem. Phys., 2003, 118, 9572-9581

65 S. Huzinaga, J. Chem. Phys., 1965, 42, 1293-1302.

66 W. Kutzelnigg, U. Fleischer and M. Schindler, Springer Verlag, Berlin, 1990, p. 165.

67 C. Wullen, Ruhr-Universität, Bochum, 1992.

68 Dalton, an ab initio electronic structure program, see http://www.daltonprogram.org.

69 R. W. Fessenden and R. H. Schuler, J. Chem. Phys., 1963, 39, $2147-2195$.

70 H. J. McManus, R. W. Fessenden and D. M. Chipman, J. Phys. Chem., 1988, 92, 3778-3781.

71 J. K. Kochi and P. J. Krusic, J. Am. Chem. Soc., 1968, 90, $7157-7159$.

72 F. Alessandro, Int. J. Quantum Chem., 1994, 52, 97-108.

73 C. Adamo, M. Cossi and V. Barone, J. Mol. Struct., 1999, 493, $145-157$.

74 S. A. Perera, L. M. Salemi and R. J. Bartlett, J. Chem. Phys., 1997, 106, 4061-4066.

75 O. B. Lutnæs, A. M. Teale, T. Helgaker, D. J. Tozer, K. Ruud and J. Gauss, J. Chem. Phys., 2009, 131, 144104.

76 P. J. Krusic, J. P. Jesson and J. K. Kochi, J. Am. Chem. Soc., 1969, 91, 4566-4568.

77 J. K. Kochi and P. J. Krusic, Essays on Free Radical Chemistry, Chem. Soc. Special Publications, 1971, 24, 147-168.

78 R. Sustmann and F. Lübbe, Tetrahedron Lett., 1974, 15, 2831-2834.

79 U. R. Böhme and H. C. Wolf, Chem. Phys. Lett., 1972, 17, $582-587$.

80 T. A. Ruden, O. B. Lutnæs, T. Helgaker and K. Ruud, J. Chem. Phys., 2003, 118, 9572.

81 A. J. Thorvaldsen, K. Ruud, K. Kristensen, P. Jørgensen and S. Coriani, J. Chem. Phys., 2008, 129, 214108. 